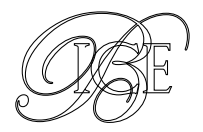

\section{LA PERCEPCIÓN DE LOS EXPORTADORES SOBRE EL APOYO A LA INTERNACIONALIZACIÓN Resultados de la EPAI 2021}

En este artículo se presentan los resultados de la Encuesta de Percepciones sobre el Apoyo a la Internacionalización de 2021 (EPAI 2021). Se trata de una investigación que aporta información sobre el conocimiento que tienen las empresas que exportan con regularidad acerca de los principales instrumentos de apoyo a la internacionalización (ICEX, CESCE, las líneas ICO de internacionalización, la red de Oficinas Económicas y Comerciales en el exterior, FIEM y COFIDES), el uso que hacen de los mismos y el grado de satisfacción con los resultados obtenidos. La EPAI 2021 ofrece, además, resultados desagregados por sectores e intervalos de exportación y, en el caso de los principales instrumentos (ICEX y CESCE), explora las causas por las que algunas empresas no los utilizan, a pesar de conocerlos. Las valoraciones de las empresas, obtenidas a través de la encuesta, facilitan que se alcancen conclusiones útiles para la mejora de la eficacia de las políticas de apoyo a la internacionalización.

Palabras clave: comercio exterior, internacionalización, pyme, exportación, ICEX, CESCE, ICO, OFECOMES.

Clasificación JEL: F13, F43, L21, L25.

\section{Introducción}

En el cuarto trimestre del año 2019 la Secretaría de Estado de Comercio realizó la Encuesta Piloto sobre Apoyo a la Internacionalización (Subdirección General de Estudios y Evaluación de Instrumentos de Política Comercial, 2020). Se ponía así en marcha un

\footnotetext{
* Secretaría de Estado de Comercio. Ministerio de Industria, Comercio y Turismo.

Versión de mayo de 2021.

DOI: https:/doi.org/10.32796/bice.2021.3135.7199
}

ejercicio orientado a mejorar el conocimiento sobre la percepción que tienen las empresas, en concreto las que exportan de forma regular, acerca de los instrumentos de apoyo a la internacionalización.

Los resultados obtenidos fueron de utilidad para la elaboración del Informe de Evaluación del Plan de Acción para la Internacionalización de la Economía Española 2017-2018 (Ministerio de Industria, Comercio y Turismo, 2021).

Sobre la base de la experiencia adquirida mediante esta encuesta piloto, en el $\triangleright$ 
primer trimestre de 2021 se ha incluido en la Encuesta de Coyuntura de la Exportación (ECE) un nuevo módulo. Este módulo ha pasado a denominarse Encuesta de Percepción sobre el Apoyo a la Internacionalización (EPAI).

El objetivo de esta investigación es contribuir a la mejora de los principales instrumentos de apoyo a la internacionalización, aportando luz sobre su visibilidad entre las empresas susceptibles de utilizar los servicios que ofrecen, el grado en que los utilizan, las razones por las que, en su caso, no lo hacen y la medida en que los resultados que obtienen satisfacen sus expectativas. Este indicador de satisfacción pretende aproximar, al menos de forma cualitativa, la eficacia percibida por las empresas sobre los apoyos recibidos.

Los resultados de la EPAI 2021 serán de utilidad para la evaluación del Plan de Acción para la Internacionalización de la Economía Española 2019-2020 (Subdirección General de Estrategia de Internacionalización, 2019). Se trata del segundo plan bienal de desarrollo de la Estrategia de Internacionalización de la Economía Española 2017-2027 (en adelante, EIEE), el marco estratégico que articula la acción pública para impulsar la internacionalización de la economía, cuyo objetivo es consolidar la contribución positiva del sector exterior al crecimiento económico e impulsar la creación de empleo (Ministerio de Economía, Industria y Competitividad, 2017; Subdirección General de Análisis y Estrategia de Internacionalización, 2019).

El primer eje de la EIEE es ofrecer un apoyo a la internacionalización adaptado al perfil de las empresas. En línea con este eje, la EPAI 2021 permite analizar cómo difieren las percepciones de las empresas sobre los instrumentos de apoyo a la internacionalización y su eficacia en función de características tales como su sector de actividad o sus volúmenes de exportación. Este análisis desagregado mejora la comprensión de la relación existente entre las características empresariales y sus necesidades en materia de internacionalización y facilita, por esta vía, un diseño más adecuado de los instrumentos de apoyo.

En suma, la EPAI 2021 pretende ser una contribución a la aplicación efectiva de los principios de transparencia y evaluación que rigen la EIEE y que son esenciales tanto para la mejor consecución de sus objetivos como para la rendición de cuentas ante la ciudadanía.

En este artículo se resumen los principales resultados de la EPAI 2021. En el epígrafe 2 se realiza una breve descripción de la metodología de la EPAI, subrayando los cambios que incorpora respecto al ejercicio piloto realizado en el último trimestre de 2019. En el epígrafe 3 se presentan los resultados sobre conocimiento, uso y satisfacción acerca de los apoyos, servicios y programas que ofrece ICEX, así como sobre las razones por las que algunas empresas no los utilizan. Se comparan estos resultados con los obtenidos en la encuesta piloto de 2019. En el epígrafe 4 se presentan los resultados para CESCE, circunscritos a su acción por cuenta del Estado. Esta opción impide una comparación con los resultados de 2019, ya que en ese año el cuestionario tenía un enfoque más general. El epígrafe 5 se dedica a presentar cómo perciben las empresas otros instrumentos de apoyo a la internacionalización de la empresa, las líneas ICO de internacionalización, la red de Oficinas Económicas y Comerciales (OFECOMES) en el exterior de la Secretaría de Estado de Comercio, el Fondo para la Internacionalización de la Empresa (FIEM) y COFIDES. En el $\triangleright$ 
último apartado se resumen las principales conclusiones.

\section{La Encuesta de Percepción sobre el Apoyo a la Internacionalización}

La EPAI 2021 supone, tras la experiencia piloto del último trimestre de 2019, la consolidación de esta herramienta de evaluación de la acción pública de apoyo a la internacionalización. Su objetivo es obtener información sobre la percepción que tienen las empresas que exportan mercancías de forma regular ${ }^{1}$ sobre los principales instrumentos de apoyo a la internacionalización: ICEX, CESCE (centrándose, en esta edición 2021, en su actuación por cuenta del Estado), las líneas ICO para la internacionalización, la red de OFECOMES, FIEM y COFIDES.

Hay que destacar que los resultados de la EPAI 2021 relativos a ICEX son los únicos que pueden compararse, de forma directa, con los obtenidos en el ejercicio piloto de 2019, ya que, con relación a otros instrumentos, o bien se ha modificado el enfoque de las preguntas (es el caso de CESCE) o bien no se recogió información en el ejercicio piloto (líneas ICO para la internacionalización, red de OFECOMES, FIEM y COFIDES).

La EPAI 2021 se apoya sobre un cuestionario muy sencillo (ver anexo) que no persigue una cuantificación precisa de la cobertura, uso

\footnotetext{
1 La definición de empresas que exportan regularmente utilizada en la ECE y en la EPAl difiere de la empleada en otros productos de la Secretaría de Estado de Comercio, como el Informe Mensual de Comercio Exterior. En el caso de la ECE y la EPAI, se trata de empresas cuya regularidad viene definida por la continuidad de exportaciones en cada uno de los años del periodo 2014-2017, exigiéndose, además, la superación anual de un umbral de exportaciones declaradas. En el caso del Informe Mensual de Comercio Exterior, se trata de operadores (no necesariamente empresas) cuya regularidad se refiere a haber declarado exportaciones en el periodo de referencia y en los tres años anteriores. No se exige la superación de un umbral.
}

o eficacia de cada uno de los apoyos, servicios o programas de apoyo a la internacionalización que ofrecen las distintas entidades o instrumentos de apoyo a la internacionalización. Se trata más bien de obtener una imagen general del grado de conocimiento que tienen las empresas que exportan regularmente acerca de estos instrumentos, el uso que hacen de ellos y el grado de satisfacción con los resultados obtenidos. Esta información es útil para responder cuestiones relacionadas con la cobertura de cada uno de los organismos y obtener una aproximación, al menos cualitativa, a su eficacia o, siendo más precisos, a la percepción que sobre su eficacia tienen sus principales beneficiarios.

La EPAI 2021 se ha realizado incorporando un módulo adicional en la Encuesta de Coyuntura de la Exportación, que trimestralmente realiza la Secretaría de Estado de Comercio del Ministerio de Industria, Comercio y Turismo. Los principales datos técnicos de esta encuesta se resumen en el recuadro. El trabajo de campo se ejecutó en el primer trimestre del año 2021.

La encuesta cubre un universo de empresas cuyas exportaciones, en el periodo 2014-2017, supusieron el $96,1 \%$ de las exportaciones de bienes españolas. Puede considerarse, por tanto, altamente representativa del volumen total de exportación de bienes. Sin embargo, no incluye a las empresas que exportan servicios, a las que exportan de forma no regular o por debajo del umbral establecido para obtener la muestra, a las que acaban de iniciarse en la exportación o aún no lo han hecho, pese a tener potencial para ello, o a las que se internacionalizan, principalmente, a través de la inversión en el exterior. Estas limitaciones de alcance deben tenerse en cuenta a la hora de valorar los resultados. 


\section{Metodología de la EPAI}

\section{Ámbito}

- Poblacional: empresas españolas que realizan de forma continua operaciones de exportación de mercancías superiores a 30.000 euros anuales.

- Temporal: se lleva a cabo con carácter trimestral. El periodo de referencia de la información habitual es el trimestre en curso, pero para el módulo EPAI 2021, información desde inicios de 2019 hasta la realización de la encuesta, y en el ejercicio piloto, desde inicios de 2017 hasta el último trimestre de 2019.

- Geográfico: el territorio del Estado español.

Unidades estadísticas: las empresas que realizan de manera continua actividades de exportación de bienes, que son asimismo las unidades informantes.

Informantes: responsables de exportación de las empresas seleccionadas.

\section{Marco y directorio}

- Se considera que una empresa tiene una actividad exportadora continua si realizó operaciones de exportación por valor superior a los 30.000 euros al año en cada uno de los años del periodo 2014-2017. Para determinar qué empresas cumplen esta condición se utiliza la información de comercio exterior de mercancías (datos de comercio declarado) del Departamento de Aduanas e Impuestos Especiales de la Agencia Estatal de la Administración Tributaria. A partir de dicha información se actualiza el directorio que sirve de base para obtener la muestra trimestral.

- El directorio consta de 25.765 empresas, que son las responsables del 96,1\% de las exportaciones españolas de mercancías para el periodo 2014-2017.

\section{Diseño muestral}

- Tamaño muestral: la muestra se compone de un total de 1.900 unidades (empresas).

- Tipo de muestreo: muestreo aleatorio estratificado.

- Estratificación: se utilizan dos variables para estratificar la población de empresas exportadoras incluidas en el directorio: sector y tamaño. Las empresas se asignan a los diferentes sectores en función del tipo de bienes que exportan (producto principal), considerando nueve sectores. La variable de tamaño utilizada es el valor anual medio de las exportaciones de las empresas en el periodo 2014-2017. Se consideran un total de cuatro categorías o tamaños. El número total de estratos considerados es, por lo tanto, de 36.

Fechas de recogida de datos: en 2019, del 19 de noviembre al 11 de diciembre; y en 2021, del 15 de febrero al 11 de marzo. 


\section{Resultados para las ayudas, programas y servicios de ICEX}

ICEX España Exportación e Inversiones (ICEX) es la entidad pública empresarial cuya misión es promover la internacionalización de las empresas españolas y la inversión extranjera en España. Con este fin desarrolla un amplio conjunto de acciones para apoyar a las empresas en su esfuerzo de internacionalización y atraer inversión extranjera de alto valor añadido.

En el periodo 2019-2020, al que se refiere el cuestionario de la EPAI 2021, la eficacia percibida de ICEX ha registrado una mejora generalizada con relación a los niveles, ya elevados, que registraba en el bienio anterior y que se reflejaron en el ejercicio piloto de 2019. Esta mejora de la eficacia que perciben los exportadores regulares es el resultado de un aumento de su conocimiento de ICEX, del uso que hacen de las ayudas, programas y servicios que ofrece, y de su satisfacción con los resultados obtenidos, que era ya muy elevada en el bienio 2017-2019 (Gráfico 1).

Como puede apreciarse en el Cuadro 1, el $22,5 \%$ de las empresas españolas que exportan regularmente ha percibido un impacto positivo de los servicios y programas de apoyo ofertados por ICEX sobre su proceso de internacionalización. Este porcentaje, referido al bienio 2019-2020, supera en 4 puntos porcentuales al de la encuesta anterior, cuyo periodo de referencia se extendía desde 2017 hasta finales de 2019, y se obtiene como resultado de calcular el porcentaje de empresas que conocen ICEX, han hecho uso de sus servicios en el bienio de referencia y se muestran satisfechas con el resultado obtenido.

Por sectores, destaca la percepción positiva en los de alimentación, bebidas y tabaco y manufacturas de consumo (32,4\% y $30,7 \%$, $\triangleright$

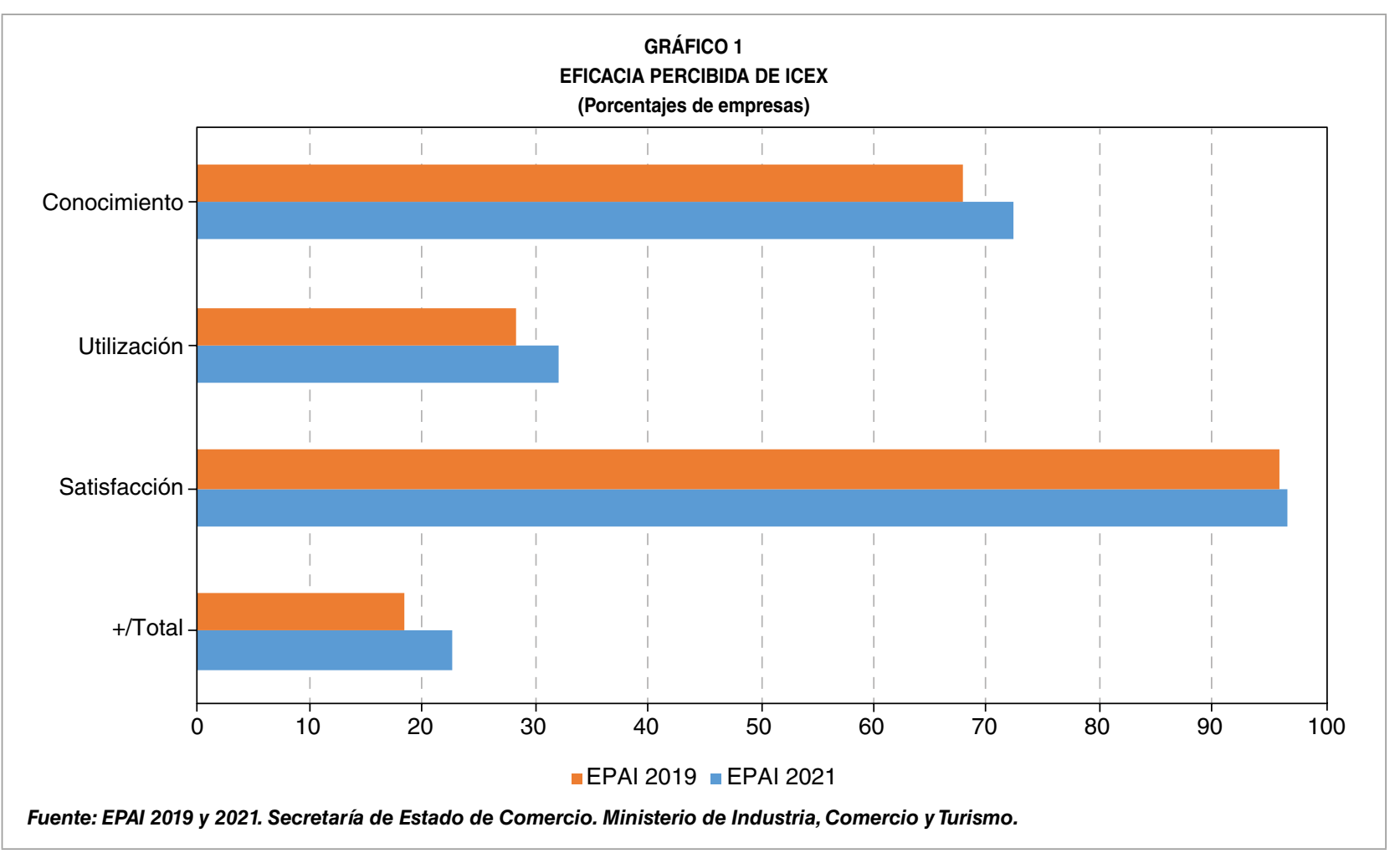


respectivamente). El impacto percibido positivo es más reducido en los sectores del automóvil y productos energéticos $(9,2 \%$ y $17,7 \%$, respectivamente), lo que posiblemente esté relacionado con las características de las empresas y del comercio en estos sectores.

Por intervalos de valor de exportación destaca, con un $23,9 \%$, el porcentaje de empresas que perciben un resultado positivo de ICEX en el intervalo medio-alto (de 3 a 15 millones de euros).

Un análisis más detallado revela que el grado de satisfacción de las empresas regulares que han utilizado los servicios de ICEX en 2019-2020 es muy elevado. Esta satisfacción es generalizada en todos los sectores e intervalos de exportación.

De forma agregada, el 96,8\% de las empresas exportadoras regulares usuarias de ICEX declara estar satisfecha con el resultado. Destaca el grado de satisfacción del $100 \%$ entre los usuarios de ICEX en los sectores de productos energéticos, materias primas, semimanufacturas no químicas y automóvil. Por intervalos de exportación, los usuarios con mayor grado de satisfacción $(97,3 \%)$ se sitúan en el de más de 15 millones de euros. El elevado grado de satisfacción es generalizado, por sectores e intervalos de tamaño, con la única excepción del sector de bienes de consumo duradero, en el que ha sido más moderado $(79,1 \%)$.

La visibilidad de ICEX también ha mejorado con relación al resultado de la encuesta piloto. El $72,3 \%$ de los exportadores regulares declara conocer ICEX. Por sectores, el grado de conocimiento de ICEX es superior al $70 \%$ en todos ellos, con las excepciones de los sectores de productos energéticos y vehículos, donde se sitúa en el $59,0 \%$ y $58,9 \%$, respectivamente. Es posible que estos resultados estén relacionados con el tipo de empresa presente en estos sectores que, por su tamaño e inserción en cadenas internacionales de valor, podrían necesitar en menor medida los servicios de ICEX. Por intervalos de exportación, la $\square$

CUADRO 1

COBERTURA Y EFICACIA DE ICEX

(Porcentaje de empresas del sector e intervalo de exportación en millones de euros)

\begin{tabular}{|c|c|c|c|c|}
\hline Sector & Conocimiento & Utilización & Satisfacción & $+/$ Total \\
\hline \multirow{5}{*}{ 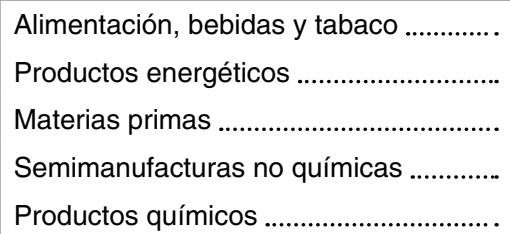 } & 84,8 & 40,5 & 94,4 & 32,4 \\
\hline & 59,0 & 30,0 & 100,0 & 17,7 \\
\hline & 76,0 & 29,8 & 100,0 & 22,7 \\
\hline & 79,1 & 26,8 & 100,0 & 21,2 \\
\hline & 71,6 & 28,0 & 98,1 & 19,7 \\
\hline 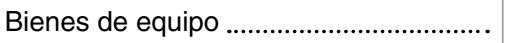 & 77,5 & 37,2 & 97,2 & 28,1 \\
\hline 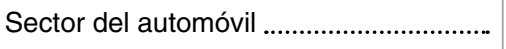 & 58,9 & 15,6 & 100,0 & 9,2 \\
\hline Bienes de consumo duradero ...................... & 71,4 & 38,5 & 79,1 & 21,7 \\
\hline Manufacturas de consumo .......................... & 70,7 & 45,6 & 95,3 & 30,7 \\
\hline \multicolumn{5}{|l|}{ Intervalo de exportación } \\
\hline 0,03 a $0,6 \ldots \ldots$. & 69,5 & 22,2 & 95,2 & 14,7 \\
\hline 0,6 a $3 \ldots \ldots$ & 76,5 & 27,6 & 93,4 & 19,8 \\
\hline 3 a 15 & 75,7 & 33,1 & 95,5 & 23,9 \\
\hline Más de 15 . & 71,5 & 32,5 & 97,3 & 22,6 \\
\hline Total . & 72,3 & 32,2 & 96,8 & 22,5 \\
\hline
\end{tabular}


CUADRO 2

RAZONES POR LAS QUE LAS EMPRESAS QUE CONOCEN ICEX NO LO UTILIZAN DESGLOSE POR SECTOR E INTERVALOS DE EXPORTACIÓN

(Porcentaje de empresas del sector e intervalo de exportación en millones de euros)

\begin{tabular}{|c|c|c|c|c|c|}
\hline Sectores & $\begin{array}{c}\text { No lo } \\
\text { necesitan }\end{array}$ & $\begin{array}{l}\text { Mejor cubierto por } \\
\text { otros organismos }\end{array}$ & $\begin{array}{c}\text { Instrumentos no adaptados } \\
\text { a las necesidades de la } \\
\text { empresa }\end{array}$ & $\begin{array}{l}\text { Requerimientos } \\
\text { de acceso } \\
\text { excesivos }\end{array}$ & $\begin{array}{l}\text { Muy } \\
\text { caro }\end{array}$ \\
\hline Alimentación, bebidas y tabaco .... & 54,8 & 6,9 & 26,0 & 7,5 & 4,9 \\
\hline Productos energéticos ......................... & 85,3 & 0,0 & 14,4 & 0,0 & 0,3 \\
\hline Materias primas & 71,7 & 5,4 & 19,6 & 1,7 & 1,7 \\
\hline Semimanufacturas no químicas ... & 57,8 & 5,0 & 31,3 & 5,1 & 0,9 \\
\hline 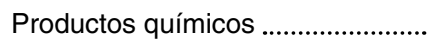 & 66,3 & 4,0 & 21,6 & 4,9 & 3,1 \\
\hline Bienes de equipo & 58,1 & 5,1 & 27,2 & 7,3 & 2,3 \\
\hline 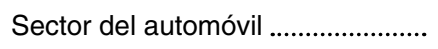 & 71,2 & 6,4 & 19,0 & 0,0 & 3,4 \\
\hline Bienes de consumo duradero ........ & 57,8 & 0,0 & 42,2 & 0,0 & 0,0 \\
\hline Manufacturas de consumo ............... & 57,8 & 5,9 & 28,2 & 6,3 & 1,9 \\
\hline \multicolumn{6}{|l|}{ Intervalos de exportación } \\
\hline 0,03 a 0,6 & 51,3 & 3,3 & 27,0 & 7,7 & 10,6 \\
\hline 0,6 a 3 & 46,8 & 6,3 & 32,0 & 10,0 & 5,0 \\
\hline 3 а 15 & 53,9 & 7,8 & 26,5 & 6,1 & 5,7 \\
\hline Más de 15 & 66,5 & 4,6 & 23,3 & 3,7 & 1,8 \\
\hline 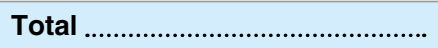 & 63,1 & 5,2 & 24,4 & 4,5 & 2,7 \\
\hline
\end{tabular}

visibilidad de ICEX es más elevada entre los exportadores regulares situados en los tramos medios (entre 0,6 y 3 millones de euros y de 3 a 15 millones de euros), situándose en el $76,5 \%$ y el $75,7 \%$, respectivamente, y algo más reducida entre los grandes exportadores (más de 15 millones), donde alcanza el 71,5\%, y entre los situados en el tramo más bajo (de 0,03 a 0,6 millones de euros), donde se sitúa en el $69,5 \%$.

El $32,2 \%$ de las empresas exportadoras regulares que conocen ICEX declara haber hecho uso de sus servicios, con porcentajes por sectores que alcanzan el 45,6 en el caso de las manufacturas de consumo y el 40,5 en el caso de alimentación, bebidas y tabaco. Por intervalos de exportación, las mayores tasas de utilización se alcanzan en los intervalos alto y medio alto. En la valoración de estos resultados debe tenerse presente, además, que la encuesta se realiza a empresas que exportan regularmente y que, por lo tanto, tienen ya una presencia consolidada en mercados exteriores, por lo que pueden no requerir, de forma continuada, de los servicios y programas de apoyo que ofrece ICEX.

Esta interpretación está alineada con los resultados que ofrece la EPAI 2021 sobre las razones por las que los exportadores regulares que conocen ICEX no han hecho uso de los servicios que ofrece en el bienio de referencia (Cuadro 2). En el 63,1\% de los casos se trata de empresas que manifiestan no tener necesidad de esos servicios. El $24,4 \%$ indica que los instrumentos ofrecidos no están adaptados a las necesidades de la empresa, mientras que solo el $5,2 \%$ declara que estas necesidades están mejor cubiertas por otros organismos. Son también minoritarios los porcentajes de exportadores regulares que aluden a requerimientos de acceso excesivos $(4,5 \%)$ o a un precio muy caro $(2,7 \%)$. 
Como se observa en el Cuadro 2, aunque existen diferencias notables entre distintos sectores e intervalos de valor de la exportación, las razones principales para no utilizar los servicios de ICEX son, en todos ellos, no necesitarlos o que no estén adaptados a las necesidades de las empresas. Cabe destacar, sin embargo, que entre los exportadores regulares incluidos en el intervalo de exportación más bajo cobran más importancia las razones asociadas al precio de los servicios y, en el intervalo medio-bajo, los requerimientos de acceso, frente a lo manifestado por los exportadores con mayor volumen de exportaciones.

\section{Resultados para la cobertura de riesgos por cuenta del Estado de CESCE}

CESCE, además de ser la cabecera de un grupo de empresas orientadas a ofrecer soluciones para la gestión del crédito comercial, es la agencia de crédito a la exportación española que gestiona el seguro de crédito a la exportación por cuenta del Estado. Este seguro de crédito cubre riesgos políticos, comerciales y extraordinarios asociados a la internacionalización de las empresas españolas.

La EPAI 2021 se circunscribe a la actividad de CESCE por cuenta del Estado, por lo que sus resultados no son comparables con los obtenidos por la EPAI 2019, cuyo cuestionario tenía un enfoque más amplio, en lo que se refiere a CESCE.

Los resultados obtenidos para el bienio 2019-2020 muestran que la actividad por cuenta del Estado ejecutada por CESCE ha tenido un impacto positivo sobre el $13,0 \%$ de las empresas españolas que exportan regularmente (Cuadro 3). Esta percepción positiva es el resultado de un grado de conocimiento de la actividad por cuenta del Estado de CESCE del $54,4 \%$, una utilización del $25,3 \%$ y un nivel de satisfacción muy elevado, en concreto del $94,3 \%$.

\section{CUADRO 3}

COBERTURA Y EFICACIA DE CESCE

(Porcentaje de empresas del sector e intervalo de exportación en millones de euros)

\begin{tabular}{|c|c|c|c|c|}
\hline Sector & Conocimiento & Utilización & Satisfacción & $+/$ Total \\
\hline \multirow{9}{*}{ 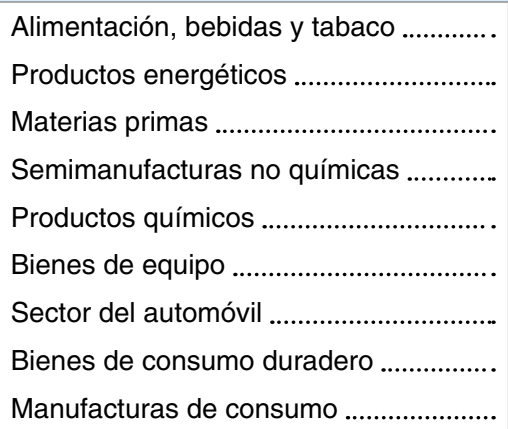 } & 61,0 & 19,0 & 97,9 & 11,3 \\
\hline & 35,4 & 33,2 & 100,0 & 11,7 \\
\hline & 58,2 & 14,7 & 91,7 & 7,9 \\
\hline & 65,3 & 31,9 & 87,9 & 18,3 \\
\hline & 52,9 & 27,0 & 91,8 & 13,1 \\
\hline & 60,0 & 25,2 & 96,9 & 14,6 \\
\hline & 44,2 & 20,2 & 100,0 & 8,9 \\
\hline & 66,9 & 16,2 & 47,1 & 5,1 \\
\hline & 53,7 & 35,0 & 93,1 & 17,5 \\
\hline \multicolumn{5}{|l|}{ Intervalo de exportación } \\
\hline 0,03 a $0,6 \ldots \ldots$ & 45,4 & 19,1 & 91,0 & 7,9 \\
\hline 0,6 a $3 \ldots \ldots$ & 52,9 & 22,9 & 86,5 & 10,5 \\
\hline 3 a 15. & 56,2 & 19,1 & 100,0 & 10,7 \\
\hline Más de 15 . & 54,2 & 26,7 & 94,0 & 13,6 \\
\hline Total. & 54,4 & 25,3 & 94,3 & 13,0 \\
\hline
\end{tabular}


CUADRO 4

RAZONES POR LAS QUE LAS EMPRESAS QUE CONOCEN CESCE NO LO UTILIZAN DESGLOSE POR SECTOR E INTERVALOS DE EXPORTACIÓN

(Porcentaje de empresas del sector e intervalo de exportación en millones de euros)

\begin{tabular}{|c|c|c|c|c|c|}
\hline Sectores & $\begin{array}{l}\text { No necesitan } \\
\text { seguro de crédito } \\
\text { a la exportación }\end{array}$ & $\begin{array}{l}\text { Mejor cubierto } \\
\text { por otras } \\
\text { entidades }\end{array}$ & $\begin{array}{c}\text { Las coberturas no se } \\
\text { adaptan a las necesidades } \\
\text { de la empresa }\end{array}$ & $\begin{array}{l}\text { Requerimientos } \\
\text { de acceso } \\
\text { excesivos }\end{array}$ & $\begin{array}{l}\text { Muy } \\
\text { caro }\end{array}$ \\
\hline Alimentación, bebidas y tabaco .... & 37,3 & 41,8 & 14,0 & 1,0 & 5,9 \\
\hline Productos energéticos ......................... & 74,8 & 0,6 & 24,6 & 0,0 & 0,0 \\
\hline Materias primas & 51,7 & 33,5 & 10,4 & 0,0 & 4,4 \\
\hline Semimanufacturas no químicas ... & 27,8 & 54,8 & 14,1 & 0,6 & 2,8 \\
\hline 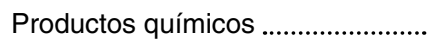 & 41,2 & 39,3 & 12,9 & 3,8 & 2,7 \\
\hline Bienes de equipo & 49,0 & 24,5 & 20,3 & 3,5 & 2,7 \\
\hline 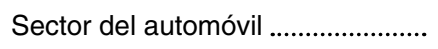 & 68,8 & 12,8 & 9,9 & 0,0 & 8,4 \\
\hline Bienes de consumo duradero ........ & 62,9 & 21,1 & 16,0 & 0,0 & 0,0 \\
\hline Manufacturas de consumo ............... & 45,1 & 30,9 & 16,4 & 4,1 & 3,5 \\
\hline \multicolumn{6}{|l|}{ Intervalos de exportación } \\
\hline 0,03 a 0,6 & 58,0 & 13,8 & 14,8 & 1,4 & 12,0 \\
\hline 0,6 a 3 & 43,5 & 26,3 & 23,9 & 3,4 & 2,8 \\
\hline 3 а 15 & 46,2 & 31,0 & 12,3 & 3,7 & 6,8 \\
\hline Más de 15 & 47,9 & 31,9 & 15,0 & 1,4 & 3,7 \\
\hline 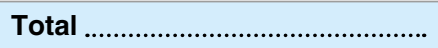 & 47,5 & 31,3 & 15,1 & 1,9 & 4,3 \\
\hline
\end{tabular}

El elevado nivel de satisfacción es generalizado por sectores, con la excepción del sector de bienes de consumo duradero, donde el indicador se sitúa en el 47,1\%. Este dato debe interpretarse con cautela, ya que un análisis más desagregado muestra que la baja satisfacción se circunscribe, dentro de este sector, al intervalo de exportaciones alto (más de 15 millones de euros), mientras que en los demás intervalos la satisfacción es plena. El peso de los exportadores en este intervalo condiciona el resultado general. En sentido positivo, destaca el grado de satisfacción del $100 \%$ en los sectores de productos energéticos y del automóvil.

Por intervalos de exportación, los niveles de satisfacción son en general elevados y oscilan entre el $86,5 \%$ en el intervalo medio bajo (de 0,6 a 3 millones de euros) y el $100,0 \%$ en el medio alto (de 3 a 15 millones de euros).

El nivel de utilización presenta cierta variación por sectores: los más elevados se presentan en manufacturas de consumo $(35,0 \%)$ y productos energéticos $(33,2 \%)$; y los más reducidos, en el de materias primas $(14,7 \%)$ y en el de bienes de consumo duradero $(16,2 \%)$. Por intervalos de exportación no se observa una pauta definida.

La visibilidad de CESCE es mayor entre los exportadores regulares de sectores como el de bienes de consumo duradero $(66,9 \%)$ y semimanufacturas no químicas $(65,3 \%)$. Las menores tasas de conocimiento se dan en el sector de productos energéticos $(35,4 \%)$ y automóvil $(44,2 \%)$.

Los resultados obtenidos invitan a examinar con mayor atención el caso del sector de bienes de consumo duradero, en el que, a pesar de una visibilidad elevada, el grado de uso es reducido y la satisfacción de los usuarios, con las cautelas de alcance de la interpretación antes señaladas, es inferior a la de otros sectores. En el mismo sentido, cabría profundizar en $\triangleright$ 
los sectores de productos energéticos y automóvil, donde la visibilidad de CESCE parece reducida, pero los niveles de utilización entre los exportadores regulares que la conocen son medios o altos y la satisfacción es muy elevada. En este tipo de sectores podría requerirse una mayor difusión de la actividad de CESCE por cuenta del Estado para potenciar los resultados positivos.

Las razones por las que las empresas que exportan regularmente no recurren a los servicios de CESCE, a pesar de conocerlos (Cuadro 4), son fundamentalmente no precisar estos servicios $(47,5 \%)$ o considerar que son mejores las coberturas ofrecidas por otras entidades $(31,3 \%)$. La falta de adaptación a las necesidades de las empresas ocupa la tercera posición, con un $15,1 \%$, mientras que son menos las empresas que indican problemas de coste de los servicios $(4,3 \%)$ o requerimientos administrativos excesivos (1,9\%).

Sectorialmente, se observan variaciones notables en la importancia relativa concedida a cada una de estas razones. En particular, destacan sectores como el de las semimanufacturas químicas o el de alimentación, bebidas y tabaco, donde la mejor cobertura ofrecida por entidades alternativas es señalada como la primera causa de no utilización, por delante de no precisar los servicios. Por intervalos de exportación, destaca la importancia que confieren los exportadores situados en el tramo bajo (entre 0,03 y 0,6 millones de euros) al coste de los servicios, frente a lo observado en intervalos mayores.

\section{Resultados para las líneas ICO para la internacionalización, la red de OFECOMES, FIEM y COFIDES}

En este epígrafe se presentan los resultados de la EPAI 2021 para otros instrumentos de apoyo a la internacionalización distintos de ICEX y CESCE. Se trata de las líneas ICO para la internacionalización, la red de OFECOMES, $\triangleright$

CUADRO 5

COBERTURA Y EFICACIA DE LAS LÍNEAS ICO DE INTERNACIONALIZACIÓN

(Porcentaje de empresas del sector e intervalo de exportación en millones de euros)

\begin{tabular}{|c|c|c|c|c|}
\hline Sector & Conocimiento & Utilización & Satisfacción & +/Total \\
\hline \multirow{9}{*}{ 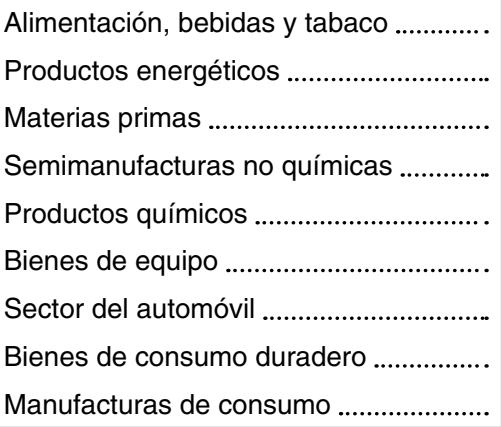 } & 74,9 & 17,8 & 94,4 & 12,6 \\
\hline & 70,2 & 8,3 & 100,0 & 5,8 \\
\hline & 75,3 & 32,5 & 100,0 & 24,5 \\
\hline & 77,7 & 17,6 & 99,8 & 13,7 \\
\hline & 65,8 & 12,2 & 100,0 & 8,0 \\
\hline & 74,3 & 22,5 & 94,6 & 15,8 \\
\hline & 72,0 & 17,5 & 87,5 & 11,1 \\
\hline & 77,2 & 21,7 & 98,8 & 16,5 \\
\hline & 70,4 & 26,6 & 97,8 & 18,3 \\
\hline \multicolumn{5}{|l|}{ Intervalo de exportación } \\
\hline \multirow{4}{*}{ 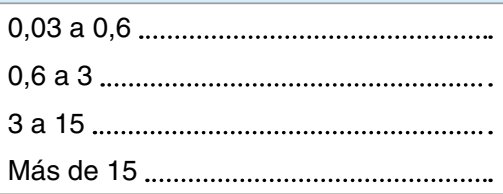 } & 72,4 & 20,9 & 89,6 & 13,6 \\
\hline & 73,8 & 26,4 & 98,7 & 19,2 \\
\hline & 74,5 & 19,8 & 96,3 & 14,2 \\
\hline & 72,0 & 17,6 & 94,6 & 12,0 \\
\hline Total & 72,5 & 18,5 & 95,2 & 12,7 \\
\hline
\end{tabular}


FIEM y COFIDES. El cuestionario utilizado para estos instrumentos es más sencillo (ver anexo) y se limita a recoger las respuestas de los exportadores regulares sobre su conocimiento de los instrumentos, su utilización y la satisfacción con el resultado obtenido.

ICO es una entidad de crédito pública que actúa como banco público de promoción del crecimiento económico y agencia financiera del Estado. En el marco de estas actuaciones, ICO dispone de una serie de productos financieros orientados a facilitar el acceso de las pymes a los mercados exteriores: ICO exportadores, ICO internacional, ICO garantías internacionales e ICO canal internacional (García de Quevedo, 2019). El cuestionario de la EPAI se refiere de forma genérica a estos productos como «líneas ICO para la internacionalización».

Los resultados de la EPAI 2021 muestran que las líneas ICO para la internacionalización han tenido un impacto positivo en el $12,7 \%$ de las empresas españolas que exportan de forma regular. Este impacto es el resultado de un nivel de visibilidad notable: el $72,5 \%$ de los exportadores regulares declara conocer estas líneas, combinada con una utilización por el $18,5 \%$ de las empresas que conoce el producto y con un nivel de satisfacción con los resultados alcanzados muy elevado, del 95,2\% (Cuadro 5).

Sectorialmente, se observa un rango variado de impactos positivos. Así, el mayor impacto positivo se percibe en el sector de materias primas, donde alcanza al $24,5 \%$ de los exportadores regulares. Por el contrario, en los sectores de productos químicos y productos energéticos se registran los impactos más reducidos (del 5,8\% y el 8,0\% de los exportadores, respectivamente).

Estos datos pueden estar relacionados con la estructura empresarial de estos sectores, dado que las líneas ICO para la internacionalización tienen por finalidad atender las necesidades de la pyme. El objetivo es paliar los fallos de mercado que, en el ámbito financiero y, en particular, en relación con la financiación de la internacionalización, afectan especialmente a este tipo de empresas. En apoyo de este razonamiento puede destacarse que los dos sectores citados registran grados bajos de utilización de estas líneas (del 12,2\% en el caso de los productos químicos y del $8,3 \%$ en el caso de los productos energéticos) y que las empresas que sí hacen uso de los instrumentos declaran una satisfacción plena (del 100,0\% en ambos casos).

Igualmente, el análisis de los resultados por intervalos del valor de la exportación muestra que las líneas ICO para la internacionalización son especialmente utilizadas por los pequeños exportadores, entre los que predominan las pymes. Así, partiendo de niveles de visibilidad del instrumento similares en los distintos intervalos de exportación, es el medio bajo (de 0,6 a 3 millones de euros) donde se registra un uso más elevado (el $26,4 \%$ de las empresas del intervalo declara haber utilizado las líneas ICO para la internacionalización en el bienio 2019-2020). En el intervalo bajo (de 0,03 a 0,6 millones) el grado de uso es del $20,9 \%$, mientras que en los intervalos superiores se sitúa por debajo del $20 \%$. Los niveles de satisfacción son elevados en todos los intervalos, aunque algo más moderados en el bajo. El resultado es un impacto positivo que alcanza su nivel más alto en el intervalo de exportación medio-bajo, donde el $19,2 \%$ de los exportadores regulares percibe un efecto positivo de estas líneas.

En suma, se trata de un instrumento con amplia visibilidad y aceptación por parte de las empresas, que genera un impacto que se percibe como positivo. Los resultados parecen $\triangleright$ 
coherentes con su orientación a paliar fallos de los mercados financieros que afectan a las pymes. Los registros, algo menos elevados, del indicador de satisfacción para los pequeños exportadores regulares requieren un análisis más en detalle para profundizar en sus causas $y$, en su caso, impulsar mejoras en su adaptación a las características de este tipo de empresas.

La red de Oficinas Económicas y Comerciales de España en el exterior (OFECOMES) dota a la Secretaría de Estado de Comercio y a sus instrumentos de apoyo a la internacionalización de una presencia activa en los principales mercados de interés para la economía española. Con esta presencia se persigue aportar a las empresas un apoyo en destino en su proceso de internacionalización.

De acuerdo con los resultados de la EPAI 2021, la red de OFECOMES ha tenido un impacto positivo para el $11,6 \%$ de las empresas españolas que exportan de forma regular. Este impacto es el resultado de un conocimiento de la red de OFECOMES por parte del $51,5 \%$ de las empresas que exportan regularmente, una utilización de este instrumento por el $24,5 \%$ de las empresas que lo conocen y un nivel de satisfacción elevado, del 91,7\% (Cuadro 6).

Por sectores, los impactos positivos más elevados se dan en el de alimentación, bebidas y tabaco $(21,1 \%)$ y bienes de equipo (18,3\%). En ambos sectores, los niveles de conocimiento, utilización y satisfacción se sitúan por encima de la media. Los impactos positivos más moderados se registran en el sector del automóvil y en el de productos energéticos. No resulta sorprendente que se trate de los mismos sectores en los que las empresas que exportan regularmente declaran impactos también moderados de los servicios de ICEX, dado que la red de OFECOMES constituye, en buena medida, un punto de apoyo en el país de destino para la prestación de estos servicios. Las consideraciones con respecto a la valoración de estos resultados, condicionados por el tamaño de las empresas y su inserción en cadenas $\triangleright$

CUADRO 6

COBERTURA Y EFICACIA DE LA RED DE OFECOMES

(Porcentaje de empresas del sector e intervalo de exportación en millones de euros)

\begin{tabular}{|c|c|c|c|c|}
\hline Sector & Conocimiento & Utilización & Satisfacción & +/Total \\
\hline \multirow{9}{*}{ 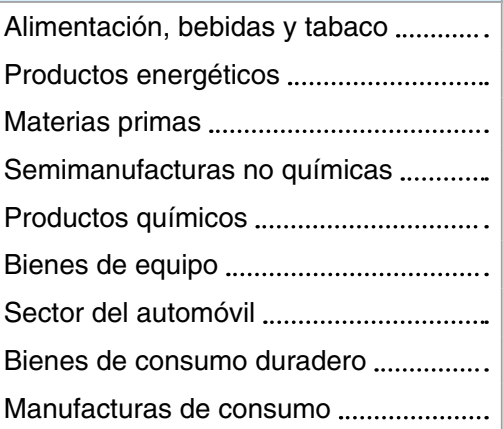 } & 60,9 & 37,3 & 93,0 & 21,1 \\
\hline & 24,0 & 25,3 & 100,0 & 6,1 \\
\hline & 52,1 & 30,1 & 71,6 & 11,2 \\
\hline & 64,0 & 11,6 & 99,6 & 7,4 \\
\hline & 53,7 & 17,4 & 95,9 & 9,0 \\
\hline & 54,4 & 35,4 & 95,0 & 18,3 \\
\hline & 44,7 & 6,6 & 50,0 & 1,5 \\
\hline & 56,8 & 39,2 & 47,6 & 10,6 \\
\hline & 45,3 & 32,9 & 99,0 & 14,8 \\
\hline \multicolumn{5}{|l|}{ Intervalo de exportación } \\
\hline \multirow{4}{*}{$\begin{array}{l}0,03 \text { a } 0,6 \\
0,6 \text { a } 3 \\
3 \text { a } 15 \\
\text { Más de } 15\end{array}$} & 39,9 & 22,4 & 91,4 & 8,2 \\
\hline & 50,8 & 22,8 & 90,0 & 10,4 \\
\hline & 55,9 & 23,8 & 97,8 & 13,0 \\
\hline & 50,9 & 24,8 & 90,7 & 11,4 \\
\hline Total. & 51,5 & 24,5 & 91,7 & 11,6 \\
\hline
\end{tabular}


internacionales de valor en ambos sectores, son también aplicables a la red de OFECOMES.

Por intervalos de exportación destaca el impacto positivo en el $13,0 \%$ de las empresas que exportan entre 3 y 15 millones de euros al año. El nivel de satisfacción con los servicios prestados es también particularmente elevado en este intervalo $(97,8 \%)$ y la visibilidad de la red de OFECOMES $(55,9 \%)$ también alcanza su máximo entre estas empresas, aunque el grado de utilización $(23,8 \%)$ es algo inferior al de los exportadores situados en el intervalo de mayor volumen.

En definitiva, los exportadores regulares manifiestan un elevado nivel de satisfacción con los servicios recibidos por la red de OFECOMES. Sin embargo, la visibilidad de la red entre estas empresas es inferior a la de otros instrumentos de vocación general, como ICEX o CESCE, o las líneas ICO de internacionalización, a pesar de que estas últimas se dirigen principalmente a pymes. El refuerzo de la visibilidad y la potenciación de la utilización, así como el análisis de la adecuación de los servicios a las características de las empresas de algunos sectores e intervalos de exportación, podrían resultar en mejoras del impacto positivo de la red.

El Fondo para la Internacionalización de la Empresa (FIEM) es un instrumento gestionado por la Secretaría de Estado de Comercio cuyo objetivo es promover la internacionalización de las empresas españolas mediante la financiación directa de sus operaciones de exportación e inversión en el exterior. Ofrece créditos a los clientes de las empresas españolas o a sus filiales extranjeras, de forma que sus proyectos disfruten de condiciones tan favorables como la de los competidores de nuestro entorno económico. En general, las operaciones FIEM conllevan un alto volumen de financiación, aunque desde 2019, en una coyuntura financiera favorable, han reforzado su orientación a las pymes a través de la línea FIEM-PYME, dedicada a financiar operaciones de exportación e inversión de este tipo de empresas y con unas condiciones financieras más flexibles y de tramitación más ágil.

La vocación más selectiva que tradicionalmente ha tenido este instrumento condiciona un impacto más reducido que el de los vehículos de orientación más general. Así, se observa un impacto positivo en el 1,0\% de los exportadores regulares que responde a una visibilidad limitada del instrumento, conocido por el 21,7\% de estas empresas, un grado de uso del $4,8 \%$ de las empresas que lo conocen y un nivel de satisfacción particularmente elevado, del 96,1\% (Cuadro 7). Al valorar este resultado debe tenerse presente, además, que FIEM se orienta tanto a financiar operaciones de exportación como proyectos de inversión y que las empresas que se internacionalizan mediante la inversión no coinciden, necesariamente, con los exportadores regulares, entre quienes se realiza la encuesta.

Los resultados sectoriales también reflejan la vocación más selectiva del instrumento, ya que, en algunos de los sectores, pese a que la visibilidad del FIEM ha sido similar a la media, no se han registrado, de acuerdo con las respuestas de las empresas, usos de este fondo. Cabe destacar que, en los sectores donde hay utilización del FIEM, el grado de satisfacción es siempre elevado. De hecho, es pleno, salvo en el sector de bienes de equipo, donde alcanza el $88,8 \%$.

Por intervalos de exportación, el instrumento ha sido más utilizado en el medio alto (de 3 a 15 millones de euros al año), donde alcanza también la mayor tasa de impacto positivo $\triangleright$ 
CUADRO 7

COBERTURA Y EFICACIA DE FIEM

(Porcentaje de empresas del sector e intervalo de exportación en millones de euros)

\begin{tabular}{|c|c|c|c|c|}
\hline Sector & Conocimiento & Utilización & Satisfacción & $+/$ Total \\
\hline Alimentación, bebidas y tabaco ................. & 19,2 & 1,7 & 100,0 & 0,3 \\
\hline 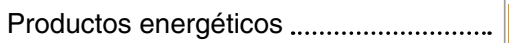 & 23,3 & 0,0 & 0,0 & 0,0 \\
\hline Materias primas & 22,7 & 0,0 & 0,0 & 0,0 \\
\hline Semimanufacturas no químicas .................. & 25,3 & 3,1 & 100,0 & 0,8 \\
\hline Productos químicos & 24,2 & 7,2 & 100,0 & 1,8 \\
\hline Bienes de equipo & 25,9 & 7,3 & 88,8 & 1,7 \\
\hline 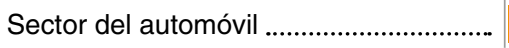 & 17,0 & 8,7 & 100,0 & 1,5 \\
\hline Bienes de consumo duradero ....................... & 10,0 & 0,0 & 0,0 & 0,0 \\
\hline Manufacturas de consumo ............................ & 19,8 & 0,0 & 0,0 & 0,0 \\
\hline \multicolumn{5}{|l|}{ Intervalo de exportación } \\
\hline 0,03 a 0,6 & 13,3 & 0,0 & 0,0 & 0,0 \\
\hline 0,6 a 3 & 16,3 & 4,2 & 100,0 & 0,7 \\
\hline 3 a 15 & 18,8 & 7,5 & 80,4 & 1,1 \\
\hline Más de 15 & 22,7 & 4,4 & 100,0 & 1,0 \\
\hline 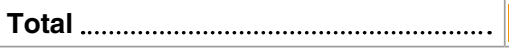 & 21,7 & 4,8 & 96,1 & 1,0 \\
\hline
\end{tabular}

$(1,1 \%)$. Sin embargo, es más conocido entre los exportadores de mayor volumen (más de 15 millones de euros al año). Estos datos apuntan a la conveniencia de continuar reforzando la línea ya emprendida en 2019 de adaptar el instrumento a las necesidades y características de las pymes, así como de potenciar su visibilidad entre este tipo de empresas. El objetivo sería continuar impulsando su papel complementario del sector privado y atender las necesidades de financiación, con relación al proceso de internacionalización, de este tipo de empresas, que son las que, en mayor medida, resultan afectadas por los fallos de mercado en el ámbito financiero.

COFIDES es una sociedad mercantil estatal cuyo objeto es facilitar financiación a proyectos privados y viables de inversión en el exterior en los que exista algún tipo de interés español (a través de la propia inversión o de exportaciones vinculadas al proyecto), para contribuir con criterios de rentabilidad tanto al desarrollo de los países receptores de las inversiones como a la internacionalización de la economía y de las empresas españolas. COFIDES utiliza, por un lado, sus propios recursos para financiar proyectos de inversión en países emergentes o en desarrollo $y$, por otro lado, gestiona por cuenta del Estado los fondos FIEX y FONPYME, creados para financiar proyectos de inversión en el exterior con independencia del grado de desarrollo del país destino del proyecto.

Al igual que en el caso de FIEM, se trata de un instrumento con vocación selectiva, lo que se refleja en un impacto positivo más reducido ( $1,5 \%$ de los exportadores regulares) que el de los instrumentos de alcance más general. Este resultado también se explica por un nivel de visibilidad algo más limitado que el de otros instrumentos (el 34,0\% de los exportadores regulares conoce COFIDES) y, sobre todo, un grado de uso inferior a la media (el $4,5 \%$ de los exportadores regulares que conoce COFIDES utiliza sus servicios). El nivel de satisfacción, por el contrario, es particularmente elevado y se sitúa en el 97,4\% (Cuadro 8). 
CUADRO 8

COBERTURA Y EFICACIA DE COFIDES

(Porcentaje de empresas del sector e intervalo de exportación en millones de euros)

\begin{tabular}{|c|c|c|c|c|}
\hline Sector & Conocimiento & Utilización & Satisfacción & $+/$ Total \\
\hline Alimentación, bebidas y tabaco ................... & 33,0 & 3,9 & 100,0 & 1,3 \\
\hline 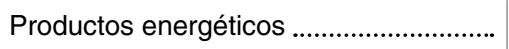 & 35,0 & 0,0 & 0,0 & 0,0 \\
\hline Materias primas & 26,3 & 0,6 & 100,0 & 0,2 \\
\hline Semimanufacturas no químicas ................. & 40,6 & 3,9 & 100,0 & 1,6 \\
\hline Productos químicos & 31,2 & 4,7 & 100,0 & 1,5 \\
\hline Bienes de equipo & 35,1 & 13,2 & 95,4 & 4,4 \\
\hline Sector del automóvil & 33,5 & 0,6 & 100,0 & 0,2 \\
\hline Bienes de consumo duradero ....................... & 20,5 & 0,0 & 0,0 & 0,0 \\
\hline Manufacturas de consumo ........................... & 33,7 & 0,4 & 100,0 & 0,1 \\
\hline \multicolumn{5}{|l|}{ Intervalo de exportación } \\
\hline 0,03 a 0,6 & 20,6 & 1,4 & 100,0 & 0,3 \\
\hline 0,6 a 3 & 24,8 & 4,6 & 100,0 & 1,1 \\
\hline 3 a 15. & 29,8 & 6,6 & 86,0 & 1,7 \\
\hline Más de $15 \ldots \ldots \ldots \ldots . . . .$. & 35,6 & 4,2 & 100,0 & 1,5 \\
\hline Total & 34,0 & 4,5 & 97,4 & 1,5 \\
\hline
\end{tabular}

El análisis sectorial revela una cierta diversificación del instrumento, a pesar de su orientación más selectiva. Los grados de conocimiento varían entre el $20,5 \%$, en el sector de bienes de consumo duradero, y el $40,6 \%$, en el de semimanufacturas no químicas. En todos los sectores, salvo en los de productos energéticos y bienes de consumo duradero, se han contabilizado usuarios de COFIDES. La satisfacción es muy elevada en todos los sectores, con niveles plenos en todos los que registran uso, salvo en el de bienes de equipo, donde se alcanza un 95,4\%.

Por intervalos de exportación, el impacto positivo más elevado se alcanza en el medio alto (de 3 a 15 millones de euros), con una repercusión favorable en el 1,7\% de las empresas que exportan regularmente dentro de este intervalo. Es también en este intervalo donde se alcanza un mayor grado de uso, aunque la satisfacción es más reducida $(86,0 \%$, frente al $100,0 \%$ registrado en otros intervalos). La visibilidad de COFIDES parece presentar cierta correlación con el volumen de exportación, es más reducida en el intervalo bajo $(20,6 \%$ ) y alcanza el nivel máximo en el más elevado $(35,6 \%)$.

\section{Conclusiones}

Los resultados de la EPAI 2021 permiten valorar cómo perciben las empresas que exportan regularmente el impacto que han tenido los principales instrumentos de apoyo a la internacionalización en el bienio 2019-2020. Dicho periodo coincide con la vigencia del segundo plan de acción bienal de aplicación de la EIEE 20172027, por lo que la EPAI 2021 resultará útil para su evaluación.

Los resultados presentados en este artículo permiten avanzar algunas conclusiones y sugerir líneas de exploración para potenciar una mayor eficacia de estos instrumentos.

En todos los casos, las empresas que exportan regularmente y utilizan los distintos $\triangleright$ 
instrumentos de apoyo muestran elevados niveles de satisfacción con los resultados obtenidos. Esta percepción sugiere que estos instrumentos son eficaces para la consecución de los objetivos de internacionalización de las empresas beneficiarias.

Con relación a ICEX, único instrumento para el que pueden compararse los resultados con los del ejercicio piloto realizado en 2019 , se observa una mejora en los indicadores de visibilidad, uso y satisfacción, que redunda en un impacto positivo más elevado. Las principales vías de mejora de este impacto serían continuar potenciando la visibilidad del instrumento y profundizar en la adaptación de los distintos apoyos, servicios y programas que ofrece ICEX a las concretas características de las empresas. El desarrollo de la estrategia de evaluación interna de ICEX y del sistema de recomendación de itinerarios de apoyo a medida para las empresas son claros avances en esta línea (Núñez y Gómez, 2020).

En el caso de CESCE, además de reforzar la visibilidad de sus actuaciones por cuenta del Estado, los resultados obtenidos sugieren la necesidad de comparar sus servicios con los ofrecidos por otras entidades, para reforzar posibles complementariedades y avanzar en aspectos en los que pueda existir margen de mejora. En este sentido, ejercicios de benchmarking con otras agencias de crédito a la exportación sugieren que es necesario dotar de mayor autonomía y flexibilidad a este instrumento (CESCE, 2020). Otro elemento a considerar es el coste de los servicios para los pequeños exportadores, que podría estar constituyendo una barrera de acceso.

Las líneas ICO para la internacionalización disfrutan, de acuerdo con los resultados de la EPAI 2021, de elevados niveles de visibilidad y aceptación. Además de continuar con la labor de difusión, parece conveniente examinar su grado de adaptación a las necesidades de los pequeños exportadores, entre los que el nivel de satisfacción es algo menos elevado.

En el caso de la red de OFECOMES, la principal recomendación que puede extraerse de los resultados de la EPAI 2021 es la de continuar potenciando su visibilidad entre los exportadores, como principal vía para aumentar su eficacia, siempre que estén dotadas con capacidad para absorber un aumento de la demanda de sus servicios, sin merma de su calidad, que, de acuerdo con los indicadores de satisfacción, se percibe como elevada.

En los instrumentos de vocación más selectiva (FIEM y COFIDES) se aprecia también un elevado nivel de satisfacción con los servicios prestados. Dado el enfoque selectivo de los instrumentos, que sus niveles de visibilidad y grado de uso sean más reducidos que los de otros instrumentos no puede considerarse, por sí mismo, un problema. De acuerdo con análisis previos, el mayor margen para la mejora de su eficacia parece residir en reforzar su orientación de complementariedad con servicios ofrecidos por el sector privado, continuando la estrategia de apertura a pymes, en el caso de FIEM, así como su coordinación con otros instrumentos de apoyo financiero a la internacionalización o de promoción del desarrollo (Ministerio de Industria, Comercio y Turismo, 2021).

\section{Bibliografía}

CESCE (2020). Agencias de crédito a la exportación. Boletín Económico de ICE, (3123). https:// doi.org/10.32796/bice.2020.3123.7002

García de Quevedo Ruiz, J. (2019). Instrumentos del Instituto de Crédito Oficial para la $\triangleright$ 


\section{LA PERCEPCIÓN DE LOS EXPORTADORES SOBRE EL APOYO A LA INTERNACIONALIZACIÓN}

financiación de la internacionalización de la empresa española. Boletín Económico de ICE, (3114). http://www.revistasice.com/index.php/ $\mathrm{BICE} /$ article/view/6889/6906

Ministerio de Economía, Industria y Competitividad (2017). La Estrategia de Internacionalización de la Economía Española 2017-2027. https://comercio.gob.es/es-es/estrategia_internacionalizacion/Paginas/Estrategia-Internacionalizacion2017-2027.aspx

Ministerio de Industria, Comercio y Turismo (2021). Informe de Evaluación del Plan de Acción para la Internacionalización de la Economía Española 2017-2018. https://comercio.gob.es/es-es/estrategia_internacionalizacion/Paginas/informe-evaluacion-plan-2017_18.aspx
Nuñez Varo, J.M., y Gómez González, M. del M. (2020). El valor estratégico de la evaluación en ICEX. Boletín Económico de ICE, (3123). https:// doi.org/10.32796/bice.2020.3123.7003

Subdirección General de Estrategia de Internacionalización (2019). El Plan de Acción para la Internacionalización de la Economía Española 2019-2020. Boletín Económico de ICE, (3111). https://doi.org/10.32796/bice.2019.3111.6827

Subdirección General de Estudios y Evaluación de Instrumentos de Política Comercial (2020). Eficacia del apoyo a la internacionalización. La percepción de los exportadores. Boletín Económico de ICE, (3123). https://doi.org/10.32796/ bice.2020.3123.7001 
Subdirección General de Estudios y Evaluación de Instrumentos de Política Comercial

\section{ANEXO \\ CUESTIONARIO EPAI 2021}

N1. ¿Conocen en su empresa la entidad ICEX España Exportación e Inversiones?

\begin{tabular}{|c|c|}
\hline Sí & Ir a la pregunta 2 \\
\hline No & Ir la pregunta 5 \\
\hline
\end{tabular}

N2. ¿Ha utilizado su empresa algún tipo de ayuda, programa o servicio de ICEX España Exportación e Inversiones desde el 1 de enero de 2019 en adelante?

\begin{tabular}{|c|c|}
\hline Sí & Ir a la pregunta N3 \\
\hline No & Ir la pregunta N4 \\
\hline
\end{tabular}

N3. ¿Quedó su empresa satisfecha con la ayuda, programa o servicio prestado por ICEX?

\begin{tabular}{|c|c|}
\hline Sí & Ir a la pregunta N5 \\
\hline No & Ir la pregunta N5 \\
\hline
\end{tabular}

N4. Señale el principal motivo por el que su empresa no ha utilizado ningún apoyo o servicio de ICEX España Exportación e Inversiones

\begin{tabular}{|l|c|}
\hline \multicolumn{1}{|c|}{ Instrumentos ICEX } & Marca \\
\hline No necesito ninguno de sus instrumentos & \\
\hline Estos instrumentos están mejor cubiertos por otro organismo & \\
\hline Los instrumentos no se adaptan a las necesidades de mi empresa & \\
\hline Los requerimientos de acceso son excesivos & \\
\hline Los servicios ofrecidos son demasiado caros & \\
\hline
\end{tabular}

N5. ¿Conocen en su empresa el seguro de crédito a la exportación e inversión exterior que gestiona CESCE por cuenta del Estado?

\begin{tabular}{|c|c|}
\hline Sí & Ir a la pregunta N6 \\
\hline No & Ir a la pregunta N9 \\
\hline
\end{tabular}

N6. ¿Ha utilizado su empresa algún tipo de cobertura de riesgos por cuenta del Estado de CESCE asociada a la exportación o inversión exterior desde el 1 de enero de 2019 en adelante?

\begin{tabular}{|c|c|}
\hline Sí & Ir a la pregunta N7 \\
\hline No & Ir a la pregunta N8 \\
\hline
\end{tabular}

N7. ¿Quedó su empresa satisfecha con el servicio prestado por CESCE?

\begin{tabular}{|c|c|}
\hline Sí & Ir a la pregunta N9 \\
\hline No & Ir a la pregunta N9 \\
\hline
\end{tabular}

N8. Señale el principal motivo por el que su empresa no ha utilizado ningún tipo de cobertura de CESCE por cuenta del Estado:

\begin{tabular}{|l|l|}
\hline \multicolumn{1}{|c|}{ Seguro de crédito a la exportación de CESCE } & Marca \\
\hline No necesito ningún seguro de crédito a la exportación & \\
\hline El seguro de crédito está mejor cubierto por otras entidades & \\
\hline Las coberturas no se adaptan a las necesidades de mi empresa & \\
\hline Los requerimientos de acceso son excesivos & \\
\hline Los servicios ofrecidos son demasiado caros & \\
\hline
\end{tabular}




\section{LA PERCEPCIÓN DE LOS EXPORTADORES SOBRE EL APOYO A LA INTERNACIONALIZACIÓN}

N9. ¿Conocen en su empresa la Compañía Española de Financiación del Desarrollo, COFIDES, S.A.?

\begin{tabular}{|c|c|}
\hline Sí & Ir a la pregunta N10 \\
\hline No & Ir a la pregunta N12 \\
\hline
\end{tabular}

N10. ¿Ha utilizado su empresa algún instrumento de capital y/o de deuda de COFIDES asociado a la exportación o inversión exterior desde el 1 de enero de 2019 en adelante?

\begin{tabular}{|c|c|}
\hline Sí & Ir a la pregunta N11 \\
\hline No & Ir a la pregunta N12 \\
\hline
\end{tabular}

N11. ¿Quedó su empresa satisfecha con el servicio prestado por COFIDES?

\begin{tabular}{|c|c|}
\hline Sí & Ir a la pregunta N12 \\
\hline No & Ir a la pregunta N12 \\
\hline
\end{tabular}

N12. ¿Conocen en su empresa el Fondo de Internacionalización de la Empresa (FIEM)?

\begin{tabular}{|c|c|}
\hline Sí & Ir a la pregunta N13 \\
\hline No & Ir a la pregunta N15 \\
\hline
\end{tabular}

N13. ¿Ha utilizado su empresa el Fondo FIEM desde el 1 de enero de 2019 en adelante?

\begin{tabular}{|c|c|}
\hline Sí & Ir a la pregunta N14 \\
\hline No & Ir a la pregunta N15 \\
\hline
\end{tabular}

N14. ¿Quedó su empresa satisfecha con la utilización de este fondo?

\begin{tabular}{|c|c|}
\hline Sí & Ir a la pregunta N15 \\
\hline No & Ir a la pregunta N15 \\
\hline
\end{tabular}

N15. ¿Conocen en su empresa las Líneas del Instituto de Crédito Oficial (ICO) para la internacionalización de la empresa?

\begin{tabular}{|c|c|}
\hline Sí & Ir a la pregunta N16 \\
\hline No & Ir a la pregunta N18 \\
\hline
\end{tabular}

N16. ¿Ha utilizado su empresa las Líneas ICO para la internacionalización desde el 1 de enero de 2019 en adelante?

\begin{tabular}{|c|c|}
\hline Sí & Ir a la pregunta N17 \\
\hline No & Ir a la pregunta N18 \\
\hline
\end{tabular}

N17. ¿Quedó su empresa satisfecha con la utilización de las Líneas ICO para la internacionalización?

\begin{tabular}{|c|c|}
\hline Sí & Ir a la pregunta N18 \\
\hline No & Ir a la pregunta N18 \\
\hline
\end{tabular}

N18. ¿Conocen en su empresa la red de oficinas económicas y comerciales de España en el exterior?

\begin{tabular}{|c|c|}
\hline Sí & Ir a la pregunta N19 \\
\hline No & FIN \\
\hline
\end{tabular}

N19. ¿Ha utilizado su empresa alguna vez los servicios de alguna oficina económica y comercial desde el 1 de enero de 2019 en adelante?

\begin{tabular}{|c|c|}
\hline Sí & Ir a la pregunta N20 \\
\hline No & FIN. \\
\hline
\end{tabular}

N20. ¿Quedó su empresa satisfecha con el servicio prestado por la oficina económica y comercial?

\begin{tabular}{|c|c|}
\hline Sí & FIN \\
\hline No & FIN \\
\hline
\end{tabular}


